\title{
Explicit Solutions for Root Optimization of a Polynomial Family
}

\author{
Vincent D. Blondel, Mert Gurbuzbalaban, Alexander Megretski and Michael L. Overton
}

\begin{abstract}
Given a family of real or complex monic polynomials of fixed degree with one fixed affine constraint on their coefficients, consider the problem of minimizing the root radius (largest modulus of the roots) or abscissa (largest real part of the roots). We give constructive methods for finding globally optimal solutions to these problems. In the real case, our methods are based on theorems that extend results in Raymond Chen's $1979 \mathrm{PhD}$ thesis. In the complex case, our methods are based on theorems that are new, easier to state but harder to prove than in the real case. Examples are presented illustrating the results, including several fixed-order controller optimal design problems.
\end{abstract}

\section{INTRODUCTION}

A fundamental general class of problems is as follows: given a set of monic polynomials of degree $n$ whose coefficients depend on parameters, determine a choice for these parameters for which the polynomial is stable, or show that no such stabilization is possible. This is a hard problem in general; indeed, it is NP-hard in certain cases [2], [12]. There is, however, one interesting special case in which the problem is directly solvable: when the dependence on parameters is affine and the number of parameters is $n-1$. In fact, in this case, the problem of globally minimizing the root radius (maximum of the moduli of the roots) or abscissa (maximum of the real parts) may be solved explicitly and efficiently. We present methods for both the real and complex cases. In the real case, our methods are based on theorems that extend results in Raymond Chen's $1979 \mathrm{PhD}$ thesis. In the complex case, our methods are based on theorems that are new, easier to state but harder to prove than in the real case. Proofs are not included in this paper but are given in [3].

\section{DisCRETE-TIME STABILITY}

Let $\rho(p)$ denote the root radius of a polynomial $p$,

$$
\rho(p)=\max \{|z|: p(z)=0, z \in \mathbb{C}\} .
$$

The following result shows that when the root radius is minimized over monic polynomials with real coefficients subject to a single affine constraint, the optimal polynomial can have at most two distinct roots (zeros). Thus, it must have at least one multiple root when $n>2$. We first state

The work of M.L. Overton was supported in part by National Science Foundation grant DMS-0714321.

V. D. Blondel, Department of Mathematical Engineering, Universit catholique de Louvain, Belgium. Email: vincent.blondel@uclouvain.be

M. Gurbuzbalaban and M. L. Overton are with the Courant Institute of Mathematical Sciences, New York University, USA. Email: mert@cims.nyu.edu, overton@cs.nyu.edu.

A. Megretski is with the Electrical Engineering Department, Massachusetts Institute of Technology, USA. Email: ameg@mit.edu. the result, and then discuss connections to previous work of Chen.

Theorem 1: Let $B_{0}, B_{1}, \ldots, B_{n}$ be real scalars (with $B_{1}, \ldots, B_{n}$ not all zero) and consider the affine family of monic polynomials

$P=\left\{z^{n}+a_{1} z^{n-1}+\ldots+a_{n-1} z+a_{n}: B_{0}+\sum_{j=1}^{n} B_{j} a_{j}=0, a_{i} \in \mathbb{R}\right\}$.

The optimization problem

$$
\rho^{*}:=\inf _{p \in P} \rho(p)
$$

has a globally optimal solution of the form

$$
p^{*}(z)=(z-\gamma)^{n-k}(z+\gamma)^{k} \in P
$$

for some integer $k$ with $0 \leq k \leq n$, where $\gamma=\rho^{*}$.

Notice that $p^{*}(z) \in P$ if and only if $\gamma$ satisfies a certain polynomial equality once $k$ is fixed. The following corollary is a direct consequence of this fact, showing that $\gamma$ in Theorem 1 can be computed explicitly.

Corollary 2: Let $\gamma$ be the the globally optimal value whose existence is asserted in Theorem 1 . Then, $-\gamma$ is a real root of smallest magnitude of the polynomials

$g_{k}(z)=B_{0} v_{0}+B_{1} v_{1} z+\ldots+B_{n-1} v_{n-1} z^{n-1}+B_{n} v_{n} z^{n}$,

where $\left(v_{0}, \ldots, v_{n}\right)$ is the convolution of the vectors

$$
\left(\left(\begin{array}{c}
n-k \\
0
\end{array}\right),\left(\begin{array}{c}
n-k \\
1
\end{array}\right), \ldots\left(\begin{array}{l}
n-k \\
n-k
\end{array}\right)\right) \text { and }\left(\left(\begin{array}{l}
k \\
0
\end{array}\right),-\left(\begin{array}{l}
k \\
1
\end{array}\right), \ldots(-1)^{k}\left(\begin{array}{l}
k \\
k
\end{array}\right)\right)
$$

for $k=0, \ldots, n$.

Theorem 1 is related to results in [8], as we now explain. Let

$$
H_{P}=\left\{\left(a_{1}, a_{2}, \ldots, a_{n}\right) \mid z^{n}+a_{1} z^{n-1}+\ldots+a_{n} \in P\right\}
$$

be the set of coefficients of polynomials in $P$. The set $H_{P}$ is a hyperplane, by which we mean an $n-1$ dimensional affine subspace of $\mathbb{R}^{n}$. Let

$$
C_{r}^{n}=\left\{\left(a_{1}, a_{2}, \ldots, a_{n}\right) \in \mathbb{R}^{n}\left|z^{n}+\sum_{j=1}^{n} a_{j} z^{n-j}=0 \Rightarrow\right| z \mid<r\right\}
$$

be the set of coefficients of monic polynomials with root radius smaller than $r$. Clearly, $\rho^{*}<r$ if and only if $H_{P} \cap C_{r}^{n} \neq \emptyset$. The root optimization problem is then equivalent to finding the infimum of $r$ such that the hyperplane $H_{P}$ intersects the set $C_{r}^{n}$. The latter set is known to be nonconvex, characterized by several algebraic inequalities, so this would appear to be difficult. However, since $C_{r}^{n}$ is open and connected, it intersects a given hyperplane if and only if its convex hull intersects the hyperplane: 
Lemma 3: (Chen [8, Lemma 2.1.2]) Let $H$ be a hyperplane in $\mathbb{R}^{n}$, that is an $n-1$ dimensional affine subspace of $\mathbb{R}^{n}$, and let $S \subset \mathbb{R}^{n}$ be an open connected set. Then $H \cap S \neq \emptyset$ if and only if $H \cap \operatorname{conv}(S) \neq \emptyset$.

The set $\operatorname{conv}\left(C_{r}^{n}\right)$ is an open simplex so it is easy to characterize its intersection with $H_{P}$ :

Theorem 4: (Chen, special case of [8, Prop. 3.1.7]; see also [10, Prop. 4.1.26] for the case $r=1$ ) We have

$$
\operatorname{conv}\left(C_{r}^{n}\right)=\operatorname{conv}\left(\nu_{1}, \nu_{2}, \ldots, \nu_{n+1}\right)
$$

where the vertices

$\nu_{k}=\left\{\left(a_{1}, a_{2}, \ldots, a_{n}\right) \in \mathbb{R}^{n} \mid(z-r)^{n-k}(z+r)^{k}=z^{n}+\sum_{j=1}^{n} a_{j} z^{j}\right\}$

are the coefficients of the polynomials $(z-r)^{n-k}(z+r)^{k}$.

Since the optimum $\rho^{*}$ is attained, the closure of $\operatorname{conv}\left(C_{\rho^{*}}^{n}\right)$ and the hyperplane $H_{P}$ must have a non-empty intersection. Theorem 1 says that, in fact, the intersection of $H_{P}$ with $C_{\rho^{*}}^{n}$ must contain at least one vertex of $\operatorname{conv}\left(C_{\rho^{*}}^{n}\right)$, and Corollary 2 explains how to find it. In contrast, Chen uses Theorem 4 to derive a procedure (his Theorem 3.2.2) for testing whether the minimal value $\rho^{*}$ of Theorem 1 is greater or less than a given value $r$. This could be used to define a bisection method for approximating $\rho^{*}$, but it would not yield the optimal polynomial $p^{*}(z)$.

Remark 5: The techniques used in Theorem 1 are all local. Thus, any locally optimal minimizer can be perturbed to yield a locally optimal minimizer of the form $(z-$ $\beta)^{n-k}(z+\beta)^{k} \in P$ for some integer $k$, where $\beta$ is the root radius attained at the local minimizer. Furthermore, all real roots $-\beta$ of the polynomials $g_{k}$ in Corollary 2 define candidates for local minimizers, and while not all of them are guaranteed to be local minimizers, those with smallest magnitude (usually there will only be one) are guaranteed to be global minimizers.

The work of Chen [8] was limited to polynomials with real coefficients. A complex analogue of Theorem 1 is simpler to state because optimizing the root radius results in a polynomial with only one distinct root, a multiple root if $n>1$. However, the proof is substantially more complicated than for the real case.

Theorem 6: Let $B_{0}, B_{1}, \ldots, B_{n}$ be complex scalars (with $B_{1}, \ldots, B_{n}$ not all zero) and consider the affine family of polynomials

$$
P=\left\{z^{n}+a_{1} z^{n-1}+\ldots+a_{n-1} z+a_{n}: B_{0}+\sum_{j=1}^{n} B_{j} a_{j}=0, a_{i} \in \mathbb{C}\right\} .
$$

The optimization problem

$$
\rho^{*}:=\inf _{p \in P} \rho(p)
$$

has an optimal solution of the form

$$
p^{*}(z)=(z-\gamma)^{n} \in P
$$

with $-\gamma$ given by a root of smallest magnitude of the polynomial

$$
h(z)=B_{n} z^{n}+B_{n-1}\left(\begin{array}{c}
n \\
n-1
\end{array}\right) z^{n-1}+\ldots+B_{1}\left(\begin{array}{c}
n \\
1
\end{array}\right) z+B_{0} .
$$

\section{Continuous-TIME STABility}

Let $\alpha(p)$ denote the root abscissa of a polynomial $p$,

$$
\alpha(p)=\max \{\operatorname{Re}(z): p(z)=0, z \in \mathbb{C}\} .
$$

We now consider minimization of the root abscissa of a monic polynomial with real coefficients subject to a single affine constraint. In this case, the infimum may not be attained.

Theorem 7: Let $B_{0}, B_{1}, \ldots, B_{n}$ be real scalars (with $B_{1}, \ldots, B_{n}$ not all zero) and consider the affine family of polynomials

$P=\left\{z^{n}+a_{1} z^{n-1}+\ldots+a_{n-1} z+a_{n}: B_{0}+\sum_{j=1}^{n} B_{j} a_{j}=0, a_{i} \in \mathbb{R}\right\}$.

Let $k=\max \left\{j: B_{j} \neq 0\right\}$. Define the polynomial of degree $k$

$h(z)=B_{n} z^{n}+B_{n-1}\left(\begin{array}{c}n \\ n-1\end{array}\right) z^{n-1}+\ldots+B_{1}\left(\begin{array}{c}n \\ 1\end{array}\right) z+B_{0}$.

Consider the optimization problem

$$
\alpha^{*}:=\inf _{p \in P} \alpha(p) .
$$

Then

$$
\alpha^{*}=\min \left\{\beta \in \mathbb{R} \mid h^{(i)}(-\beta)=0 \text { for some } i \in\{0, \ldots, k-1\}\right\},
$$

where $h^{(i)}$ is the $i$-th derivative of $h$. Furthermore, the optimal value is attained by a minimizing polynomial $p^{*}$ if and only if $-\alpha^{*}$ is a root of $h$, that is $i=0$, and in this case we can take

$$
p^{*}(z)=(z-\gamma)^{n} \in P
$$

with $\gamma=\alpha^{*}$.

The first part of this result, the characterization of the infimal value, is due to Chen [8, Theorem 2.3.1]. Furthermore, he also observed the "if" part of the second statement, showing [8, p.29] that if $-\alpha^{*}$ is a root of $h$ (as opposed to one of its derivatives), the optimal value $\alpha^{*}$ is attained by the polynomial with a single distinct root $\alpha^{*}$. However, he noted on the same page that he did not have a general method to construct a polynomial with an abscissa equal to a given value $\tilde{\alpha}>\alpha^{*}$. Nor did he characterize the case when the infimum is attained. As in the root radius case, two roots play a role, but here one of them may not be finite. More specifically, the infimum of the root abscissa may be arbitrarily well approximated by a polynomial with two distinct roots, only one of which is bounded, as we explain in the next theorem.

Theorem 8: Assume that $-\alpha^{*}$ is not a root of $h$. Let $l$ be the smallest integer $i \in\{1, \ldots, k-1\}$ for which $-\alpha^{*}$ is a root of $h^{(i)}$. Then, for all sufficenty small $\epsilon>0$ there exists a real scalar $M_{\epsilon}$ for which

$$
p_{\epsilon}(z):=\left(z-M_{\epsilon}\right)^{m}\left(z-\left(\alpha^{*}+\epsilon\right)\right)^{n-m} \in P
$$

where $m=l$ or $l+1$, and $M_{\epsilon} \rightarrow-\infty$ as $\epsilon \rightarrow 0$.

Remark 9: If $-\beta$ is a real root of $h(z)$, then $(z-\beta)^{n} \in P$. Such a polynomial is usually, though not always, a local 
minimizer of $\alpha(p)$, but it is a global minimizer if and only if $-\beta$ is the largest such real root and no other roots of derivatives of $h$ are larger than $-\beta$.

Now we consider the optimal abscissa problem with complex coefficients. In this case, the infimal value is always attained at a polynomial with a single distinct root. Theorem 7 shows that in the real case the infimal value is not attained if and only if the polynomial $h$ has a derivative with a real root to the right of the rightmost real root of $h$. However, it is not possible that a derivative of $h$ has a complex root to the right of the rightmost complex root of $h$. This follows immediately from the Gauss-Lucas theorem, which states that the roots of the derivative of a polynomial $p$ must lie in the convex hull of the roots of $p$ [6], [11].

Theorem 10: Let $B_{0}, B_{1}, \ldots, B_{n}$ be complex scalars (with $B_{1}, \ldots, B_{n}$ not all zero) and consider the affine family of polynomials

$P=\left\{z^{n}+a_{1} z^{n-1}+\ldots+a_{n-1} z+a_{n}: B_{0}+\sum_{j=1}^{n} B_{j} a_{j}=0, a_{i} \in \mathbb{C}\right\}$.

The optimization problem

$$
\alpha^{*}:=\inf _{p \in P} \alpha(p)
$$

has an optimal solution of the form

$$
p^{*}(z)=(z-\gamma)^{n} \in P
$$

with $\gamma$ given by a root with smallest real part of the polynomial $h(-z)$ where

$h(z)=B_{n} z^{n}+B_{n-1}\left(\begin{array}{c}n \\ n-1\end{array}\right) z^{n-1}+\ldots+B_{1}\left(\begin{array}{l}n \\ 1\end{array}\right) z+B_{0}$.

\section{EXAMPLES}

Example 1. Our first example is from [5], where it was proved using the Gauss-Lucas Theorem that $p_{*}(z)=z^{n}$ is a global optimizer of the abscissa over the set of polynomials

$P=\left\{z^{n}+a_{1} z^{n-1}+\ldots+a_{n-1} z+a_{n} \mid a_{1}+a_{2}=0, a_{i} \in \mathbb{C}\right\}$.

We calculate $h(z)=\left(\begin{array}{l}n \\ 2\end{array}\right) z\left(z+\frac{2}{n-1}\right)$. Theorem 7 proves global optimality over $a_{i} \in \mathbb{R}$ and Theorem 10 proves global optimality over $a_{i} \in \mathbb{C}$.

Example 2. Consider the problem of finding a fixed-order linear controller that maximizes the closed-loop asymptotic decay rate for the classical two-mass-spring system. Henrion and Overton [9] showed that the only order for which there is a nontrivial solution is 2 , because an order 3 controller can achieve an arbitrarily fast decay rate, while using order 1 the system is not stabilizable. In the case of order 2 , the problem is equivalent to the following optimization problem [9]

$$
\inf _{p \in P} \alpha(p)
$$

where

$P=\left\{\left(s^{4}+2 s^{2}\right)\left(x_{0}+x_{1} s+s^{2}\right)+y_{0}+y_{1} s+y_{2} s^{2} \mid x_{0}, x_{1}, y_{0}, y_{1}, y_{2} \in \mathbb{R}\right\}$.

Thus $P$ is a set of monic polynomials with degree 6 whose coefficients depend affinely on 5 parameters, or equivalently with a single affine constraint on the coefficients. Motivated by numerical experiments, Henrion and Overton [9] constructed a polynomial with one distinct root with multiplicity 6 and proved its local optimality using techniques from nonsmooth analysis. Theorem 7 proves its global optimality.

Example 3. This is derived from a "Belgian chocolate" stabilization challenge problem of Blondel [1]: given $a(s)=$ $s^{2}-2 \delta s+1$ and $b(s)=s^{2}-1$, find the range of real values of $\delta$ for which there exist polynomials $x$ and $y$ such that $\operatorname{deg}(x) \geq \operatorname{deg}(y)$ and $\alpha(x y(a x+b y))<0$. This problem remains unsolved. However, inspired by numerical experiments, [4] gave a solution for $\delta<\bar{\delta}=(1 / 2) \sqrt{2+\sqrt{2}} \approx$ 0.924 . When $x$ is constrained to be a monic polynomial with degree 3 and $y$ to be a constant, the minimization of $\alpha(x y(a x+b y))$ reduces to

$$
\inf _{p \in P} \alpha(p)
$$

where

$P=\left\{\left(s^{2}-2 \delta s+1\right)\left(s^{3}+\sum_{k=0}^{2} w_{k} s^{k}\right)+\left(s^{2}-1\right) v \mid w_{0}, w_{1}, w_{2}, v \in \mathbb{C}\right\}$.

For nonzero fixed $\delta, P$ is a set of monic polynomials with degree 5 whose coefficients depend affinely on 4 parameters, or equivalently with a single affine constraint on the coefficients. In [4] a polynomial in $P$ with a distinct root of multiplicity 5 was constructed and proved to be locally optimal using nonsmooth analysis. Theorem 7 proves its global optimality over $a_{i} \in \mathbb{R}$ and Theorem 10 proves its global optimality over $a_{i} \in \mathbb{C}$. They also apply to the case when $x$ is constrained to be monic with degree 4; then, as shown in [4], stabilization is possible for $\delta<\tilde{\delta}=$ $(1 / 4) \sqrt{10+2 \sqrt{5}} \approx 0.951$.

Example 4. The polynomial achieving the minimal root radius may not be unique. Let $P=\left\{z^{2}+a_{1} z+a_{2} \mid 1+\right.$ $\left.a_{1}+a_{2}=0, a_{i} \in \mathbb{R}\right\}$. We have

$\rho^{*}=\inf _{a_{2} \in \mathbb{R}} \rho\left(z^{2}-\left(a_{2}+1\right) z+a_{2}\right)=\inf _{a_{2} \in \mathbb{R}} \rho\left(\left(z-a_{2}\right)(z-1)\right)=1$.

The minimal value is attained on a continuum of polynomials of the form $\left(z-a_{2}\right)(z-1)$ for any $-1 \leq a_{2} \leq 1$ and hence minimizers are not unique. The existence of the minimizers $(z-1)^{2}$ and $(z+1)(z-1)$ is consistent with Theorem 1 . The same example shows that the minimizer for the radius optimization problem with complex coefficients may not be unique.

Example 5. Likewise, a polynomial achieving the minimal root abscissa may not be unique. Let $P=\left\{z^{2}+a_{1} z+\right.$ $\left.a_{2} \mid a_{1}=0, a_{2} \in \mathbb{R}\right\}$. We have

$$
\alpha^{*}=\inf _{p \in P} \alpha(p)=\inf _{a_{2} \in \mathbb{R}} \alpha\left(z^{2}+a_{2}\right)=0 .
$$

Here $B_{0}=B_{2}=0, B_{1}=1$. The optimum is attained at $p^{*}(z)=z^{2}$, where $-\alpha^{*}=0$ is a root of the polynomial $h(z)=z$, as claimed in Theorem 7. However, the optimum is attained at a continuum of polynomials of the form $z^{2}+a_{2}$ for any $a_{2}>0$. 


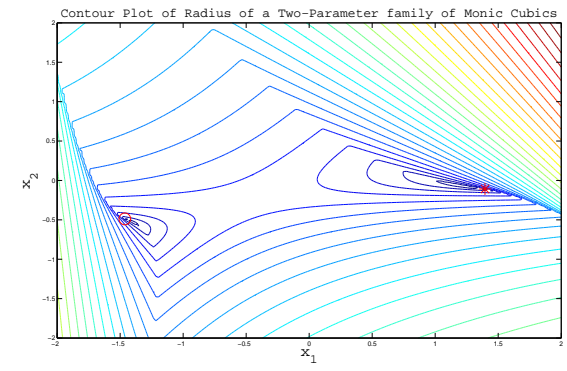

Fig. 1. Contour plot $\rho(p)$ for a randomly generated family of monic cubics with coefficients depending affinely on two real parameters. The asterisk (right) shows the global minimizer and the circle (left) shows a local minimizer.

Example 6. In this example, the infimal root abscissa is not attained. Let $P=\left\{z^{2}+a_{1} z+a_{2} \mid a_{2}=-1, a_{1} \in \mathbb{R}\right\}$

$$
\begin{aligned}
\alpha^{*}= & =\inf _{a_{1} \in \mathbb{R}} \alpha\left(z^{2}+a_{1} z-1\right) \\
& =\inf _{a_{1} \in \mathbb{R}} \max \left\{\frac{-a_{1}-\sqrt{a_{1}^{2}+4}}{2}, \frac{-a_{1}+\sqrt{a_{1}^{2}+4}}{2}\right\} \\
& =0 .
\end{aligned}
$$

This infimum is not attained, but as $a_{1} \rightarrow \infty$, setting $\epsilon=$ $\frac{-a_{1}+\sqrt{a_{1}^{2}+4}}{(z-\epsilon)\left(z-M_{\epsilon}\right)} \rightarrow 0$ and $M_{\epsilon}=\frac{-a_{1}-\sqrt{a_{1}^{2}+4}}{2} \rightarrow-\infty$ as claimed in Theorem 8.

Example 7. Figure 1 shows a contour plot of $\rho(p)$ for a randomly generated family of monic cubics with coefficients depending affinely on two real parameters. The asterisk indicates a global minimizer $\left(z-\gamma_{1}\right)^{3}$ and the circle indicates a local minimizer $\left(z-\gamma_{2}\right)^{3}$. Both $-\gamma_{1} \approx-0.541$ and $-\gamma_{2} \approx 0.567$ are roots of $g_{0}$ (see Corollary 2 and Remark 5). Note the steep contours near the global and local minimizers, indicating the non-Lipschitz behavior of the radius $\rho$ as the triple root splits [7].

Example 8. Consider the monic quintic polynomials subject to the affine constraint $B_{0}+\sum_{i=1}^{5} a_{i}=0$. Figure 2 plots the optimal abscissa, over $a_{i} \in \mathbb{R}$ and $a_{i} \in \mathbb{C}$ respectively, as a function of $B_{0} \in[-2,4]$. For $B_{0} \leq 1$, the largest root of the polynomial $h$ is real so the optimal abscissa is attained with the same optimal value for both the real and complex cases. For $B_{0}=1, h(z)=(1+z)^{5}$ so all of the roots of $h$ and its derivatives are identical. Furthermore, when $B_{0}=1$, the hyperplane $H_{P}=\left\{\left(a_{1}, a_{2}, \ldots, a_{n}\right) \mid B_{0}+\sum_{i=1}^{5} a_{i}=\right.$ $0\}$ contains the extreme lines $L_{i}$ of the cone $\operatorname{conv}\left(S_{1}^{n}\right)$ for $i=1,2,3,4$ as well as the boundary of $\operatorname{conv}\left(S_{1}^{n}\right)$ which is the positive span of the vectors $L_{i}, i=1,2,3$, 4 , we have $\operatorname{aff}\left(S_{1}^{n}\right)=H_{P}$. As $B_{0}$ is increased, the hyperplane $H_{P}$ is translated away from $\operatorname{conv}\left(S_{1}^{n}\right)$, and for $B_{0}>1, H_{P}$ does not intersect $\operatorname{conv}\left(S_{1}^{n}\right)$ but it is parallel to it. Thus, for the real case, the infimum is not attained for $B_{0}>1$ and has the constant value 1 .

More examples may be explored by downloading a publicly available ${ }^{1}$ MATLAB code implementing the constructive

\footnotetext{
${ }^{1}$ www.cs.nyu.edu/overton/software/affpoly
}

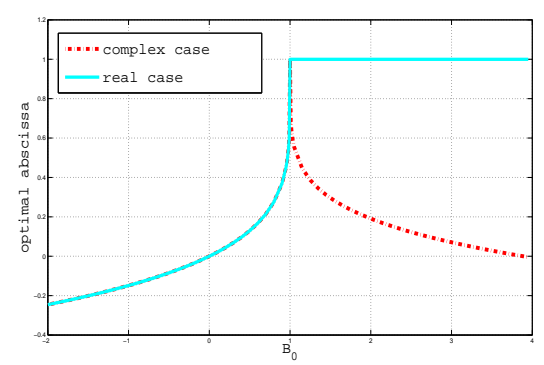

Fig. 2. The optimal root abscissa for a polynomial family $z^{5}+a_{1} z^{4}+$ $\cdots+a_{5}$ with constraint $B_{0}+\sum_{i=1}^{5} a_{i}=0$, with $B_{0}$ varied from -2 to 4. The solid curve (cyan) shows the optimal abscissa for the case $a_{i} \in \mathbb{R}$ and the dotted curve (red) for the case $a_{i} \in \mathbb{C}$.

algorithms implicit in Theorems 1, 6, 7 and 10. This code was used to generate the optimal values plotted in Figure 2 and the global and local minimizers plotted in Figure 1; see the website for more details. In general, there does not seem to be any difficulty obtaining an accurate globally optimal value for the abscissa or radius in the real or complex case. However, even in the cases where an optimal solution exists, the coefficients may be large, so that rounding errors in the computed coefficients result in a large constraint residual. Furthermore, the multiple roots are not robust with respect to small perturbations in the coefficients. Optimizing the complex stability radius of the polynomial may be of more practical use; see [4, Section II].

Acknowledgement. We thank A. Rantzer for bringing Chen's work to our attention.

\section{REFERENCES}

[1] V. Blondel. Simultaneous Stabilization of Linear Systems. Lecture Notes in Control and Information Sciences 191. Springer, Berlin, 1994.

[2] V. Blondel and J.N. Tsitsiklis. NP-hardness of some linear control design problems. SIAM Journal on Control and Optimization, 35:21182127, 1997.

[3] V.D. Blondel, M. Gurbuzbalaban, A. Megretski, and M.L. Overton. Explicit solutions for root optimization of a polynomial family with one affine constraint. 2010. Submitted for publication.

[4] J.V. Burke, D. Henrion, A.S. Lewis, and M.L. Overton. Stabilization via nonsmooth, nonconvex optimization. IEEE Transactions on Automatics Control, 51:1760-1769, 2006.

[5] J.V. Burke, A.S. Lewis, and M.L. Overton. Optimizing matrix stability. Proceedings of the American Mathematical Society, 129:1635-1642, 2001.

[6] J.V. Burke, A.S. Lewis, and M.L. Overton. Variational analysis of the abscissa mapping for polynomials via the Gauss-Lucas theorem. Journal of Global Optimization, 28:259-268, 2004.

[7] J.V. Burke, A.S. Lewis, and M.L. Overton. Variational analysis of functions of the roots of polynomials. Mathematical Programming, 104:263-292, 2005.

[8] R. Chen. Output Feedback Stabilization of Linear Systems. PhD thesis, University of Florida, 1979.

[9] D. Henrion and M. L. Overton. Maximizing the closed loop asymptotic decay rate for the two-mass-spring control problem. Technical Report 06342, LAAS-CNRS, March 2006. http://homepages.laas.fr/henrion/Papers/massspring.pdf.

[10] D. Hinrichsen and A.J. Pritchard. Mathematical Systems Theory I: Modelling, State Space Analysis, Stability and Robustness. Springer, Berlin, Heidelberg and New York, 2005.

[11] M. Marden. Geomotry of Polynomials. American Mathematical Society, 1966.

[12] A. Nemirovskii. Several NP-hard problems arising in robust stability analysis. Math. Control Signals Systems, 6:99-105, 1993. 